\title{
Brain changes associated with cognitive and emotional factors in chronic pain: A systematic review
}

\author{
A. Malfliet ${ }^{1,2,3,4}$, I. Coppieters ${ }^{2,4}$, P. Van Wilgen ${ }^{1,2,5}$, J. Kregel ${ }^{2,4}$, R. De Pauw ${ }^{4}$, M. Dolphens ${ }^{4}, \mathrm{~K}$. \\ Ickmans $s^{1,2,3}$ \\ 1 Department of Physiotherapy, Human Physiology and Anatomy (KIMA), Faculty of Physical Education and Physiotherapy, Vrije Universiteit \\ Brussels, Belgium \\ 2 Pain in Motion International Research Group, Brussels, Belgium \\ 3 Department of Physical Medicine and Physiotherapy, University Hospital Brussels, Belgium \\ 4 Department of Rehabilitation Sciences and Physiotherapy, Faculty of Medicine and Health Sciences, Ghent University, Belgium \\ 5 Transcare, Transdisciplinary Pain Management Centre, Groningen, The Netherlands
}

\begin{abstract}
Correspondence
Anneleen Malfliet

E-mail: anneleen.malfliet@vub.be

\section{Funding sources}

Anneleen Malfliet is a PhD research fellow

funded by the Research Foundation Flanders

(FWO), Belgium. Jeroen Kregel is a PhD

research fellow of the Agency for Innovation

by Science and Technology (IWT) - Applied

Biomedical Research Program (TBM), Bel-

gium. Iris Coppieters is a PhD student,

funded by the special research fund of

Ghent University (BOF Ghent). Mieke Dol-

phens and Kelly Ickmans are both postdoc-

toral research fellows of the Agency for

Innovation by Science and Technology (IWT)

- Applied Biomedical Research Program

(TBM), Belgium.
\end{abstract}

Conflicts of interest

None declared.

Systematic Review Registration Number: PROSPERO - CRD42015029454

Accepted for publication

2 December 2016

doi:10.1002/ejp.1003

\begin{abstract}
An emerging technique in chronic pain research is MRI, which has led to the understanding that chronic pain patients display brain structure and function alterations. Many of these altered brain regions and networks are not just involved in pain processing, but also in other sensory and particularly cognitive tasks. Therefore, the next step is to investigate the relation between brain alterations and pain related cognitive and emotional factors. This review aims at providing an overview of the existing literature on this subject. Pubmed, Web of Science and Embase were searched for original research reports. Twenty eight eligible papers were included, with information on the association of brain alterations with pain catastrophizing, fear-avoidance, anxiety and depressive symptoms. Methodological quality of eligible papers was checked by two independent researchers. Evidence on the direction of these associations is inconclusive. Pain catastrophizing is related to brain areas involved in pain processing, attention to pain, emotion and motor activity, and to reduced top-down pain inhibition. In contrast to pain catastrophizing, evidence on anxiety and depressive symptoms shows no clear association with brain characteristics. However, all included cognitive or emotional factors showed significant associations with resting state fMRI data, providing that even at rest the brain reserves a certain activity for these pain-related factors. Brain changes associated with illness perceptions, pain attention, attitudes and beliefs seem to receive less attention in literature.

Significance: This review shows that maladaptive cognitive and emotional factors are associated with several brain regions involved in chronic pain. Targeting these factors in these patients might normalize specific brain alterations.
\end{abstract}

\section{Introduction}

Chronic pain is characterized by a high prevalence and a tremendous personal impact, resulting in long-term sick leave, low quality of life and high socioeconomic costs (Cabral et al., 2014; Diaz
Mohedo et al., 2014; Dureja et al., 2014; Hauser et al., 2014; Jackson et al., 2014; Riskowski, 2014; Vehof et al., 2014). To influence these negative consequences, it is necessary to gain insight into the 
underlying mechanisms of chronic pain and to find a way to transfer this knowledge into clinical practice. One of the most important emerging techniques of the last decade in this field is Magnetic Resonance Imaging (MRI; Schmidt-Wilcke, 2015), which has led to the understanding that chronic pain patients display brain alterations regarding brain function and structure (Apkarian et al., 2004; Foerster et al., 2012; Ichesco et al., 2014; Lieberman et al., 2014). In addition, these neuroplastic changes are shown to be related to the persistence of pain, long after the initial nociceptive input has disappeared (Apkarian et al., 2005). Even more importantly, there is abundant evidence for a positive association between pain duration and the extent of measured brain alterations, implicating that the longer pain continues, the more the brain changes (Obermann et al., 2013; Yu et al., 2014).

Besides its association with brain changes, pain duration and thus chronification is related to maladaptive cognitive and emotional components, like pain catastrophizing, kinesiophobia (fear of movement or injury), maladaptive pain perceptions etc. (Bigos et al., 1992; Burton et al., 1995; Apkarian et al., 2005; Wiech et al., 2008; Wiech and Tracey, 2009). It is described that pain catastrophizing for example, may account for $47 \%$ of the variance in predicting the development of chronic pain after an episode of acute pain (Burton et al., 1995). Pain catastrophizing can be defined as the tendency to describe a pain experience in more exaggerated terms, and can comprise ruminating about pain, magnification of pain and/or feeling helplessness regarding pain.

As many brain regions and networks involved in the processing of pain are also involved in other sensory and particularly cognitive tasks, the next step is to take a closer look at the relation between these brain alterations and pain related cognitive and emotional factors in patients with chronic pain. Pain rumination for instance, involves excessive attention to distressing thoughts and stimuli and could therefore affect brain networks associated with attention, pain perception and pain modulation. Furthermore, a meta-analysis of imaging studies in anxiety disorders showed greater activation in specific brain areas related to fear in patients with certain phobias (Etkin and Wager, 2007).

Although there are multiple brain imaging studies looking at the relation between brain alterations and pain related cognitive and emotional factors in different chronic pain populations (Gracely et al., 2004; Geha et al., 2008; Cifre et al., 2012), currently no clear overview exists on how brain alterations are related to cognitive/emotional factors of pain in the general chronic pain population. Therefore, the aim of this systematic review is to explore and examine the existing literature on the relation between maladaptive cognitive and emotional factors related to pain and brain alterations measured by MRI in chronic pain patients.

\section{Methods}

\subsection{Registration}

This systematic review was conducted in accordance with the PRISMA (Preferred Reporting Items for Systematic reviews and Meta-Analyses) guidelines (Moher et al., 2009). The review protocol is listed in the PROSPERO register under the registration number CRD42015029454 and can be accessed online (http://www.crd.york.ac.uk/PROSPERO/display_record. asp? ID $=$ CRD42015029454).

\subsection{Literature search strategy}

A systematic search of existing, relevant literature was performed by screening Pubmed, Web of Science, and Embase. These databases were searched until October 16th, 2015.

The search strategy was based on the PICO-framework (Patient $=$ chronic pain patients; Instrument of measurement $=$ self-reported questionnaires and MRI; Comparison = not applicable; Outcome $=$ maladaptive pain related cognitive and emotional factors and brain alterations); and used search terms are listed in Table 1. The construct of the search strategy is presented in the Supporting Information Appendix S1. In addition, reference lists of relevant published articles were searched.

\subsection{Study selection - Eligibility criteria}

To be included in the present systematic review, articles had to be full-text reports or original research (no abstracts, case reports, reviews, meta-analysis, letters or editorials) written in English, French, Dutch or German. Furthermore, they had to report results on the association between maladaptive painrelated cognitive and emotional factors $(\mathrm{O})$ measured by self-reported questionnaires (I) and brain alterations (O) measured by MRI (I) in adult people with chronic pain (pain being present for at least 3 months; P).

All titles and abstracts were checked to identify relevant articles. Articles not fulfilling each of the above mentioned criteria were excluded, as well as 
Table 1 Search terms.

\begin{tabular}{|c|c|c|c|c|}
\hline \multicolumn{2}{|c|}{$\begin{array}{l}\text { Maladaptive pain-related cognitive and } \\
\text { emotional processes }\end{array}$} & \multicolumn{2}{|l|}{ Brain alterations } & \multirow{2}{*}{$\begin{array}{l}\text { Chronicity } \\
\text { Chronic Pain }\end{array}$} \\
\hline Kinesiophobia & Fear of Movement & Magnetic Resonance Imaging & Diffusion Magnetic Resonance Imaging & \\
\hline Fear of Pain & Catastrophization & rs-fMRI & Resting state fMRI & Intractable Pain \\
\hline Pain Cognitions & Pain Perception & VBM & voxel-based-morphometry & \\
\hline Ilness Perception & Anxiety & SBM & Surface based morphometry & \\
\hline Pain Attention & Psychosocial & Diffusion Tensor Imaging & Neuroimaging & \\
\hline Psychological & Attitude & Functional connectivity & Structural connectivity & \\
\hline Beliefs & & Brain morphometry & & \\
\hline
\end{tabular}

articles including persons with traumatic brain injury, migraine and/or people being over 70 years old. Additionally, this review focused only on maladaptive pain-related cognitive and emotional factors, meaning that personal characteristics (e.g. neuroticism) or executive function (e.g. attention, task switching etc.) were not of interest.

After screening on title and abstract, potentially eligible articles were retrieved in full text and were evaluated again on meeting the inclusion criteria. In case the abstract did not provide sufficient information, the full text was also retrieved. An article was included in the present systematic review when it complied with all selection criteria.

\subsection{Risk of bias}

Literature was searched and screened by AM who has experience performing a systematic review (Malfliet et al., 2015). Methodological quality was separately assessed by two independent, blinded researchers (AM and IC), who were not acquainted with each other's evaluation of the search results. After rating the selected articles, the results of both researchers were compared and differences were analysed in a consensus meeting. In case of disagreement, the reviewers screened the manuscript again and the points of difference were discussed. Reviewers achieved consensus through discussion. When consensus could not be reached, a third decisive opinion was provided by a third researcher (RDP). The methodological quality was evaluated by using the Effective Public Health Practice Project (EPHPP) Quality Assessment Tool, which has an excellent inter-rater agreement for the final score (http:// www.city.hamilton.on.ca/phcs/EPHPP/; Armijo-Olivo et al., 2012) and has been reported to have sufficient content and construct validity (Thomas et al., 2004; Jackson and Waters, 2005). This tool can be used to evaluate a variety of study designs such as randomized controlled trials (RCT) and case-control studies. The EPHPP assesses six domains, including selection bias, study design, confounders, blinding, data collection method, and withdrawals/dropouts. Each domain has two to three items that can be rated as strong ( 3 points), moderate ( 2 points) or weak (1 point), and the item scores are averaged to provide the total score of the specific domain. The maximum total score for a domain is 3.00; and based on this total score, domains are considered strong (2.51$3.00)$, moderate (1.51-2.50) or weak (1.00-1.50). A general quality rating is given to each study (strong, moderate of weak), depending on the quality of each specific domain.

To comply with the nature of imaging studies, the EPHPP was modified by replacing items 'Intervention Integrity' $(\mathrm{G})$ and 'Analyses' $(\mathrm{H})$ by a new item on MRI data quality and pre-processing. This new item comprised two elements, one focusing on the description of visual inspection of the MRI data quality' (Yes/No/Can't tell) and one checking 'if manual exclusion in case of low data quality was implemented and/or data adjustment was included in the pre-processing pipeline' (Yes/No/Can't tell). This 'MRI' item was given a final score, being strong (two times Yes), moderate (one time Yes, one time No/ Can't tell), or weak (two times No/Can't tell) and this final score was taken into account in the global rating of methodological quality of each included study.

Based on study design and methodological quality, each individual study was given a level of evidence, according to the 2005 classification system of the Dutch Institute for Healthcare Improvement CBO ("Supporting Information Table S2").

\subsection{Data collection}

All data regarding the association between maladaptive pain related cognitive/emotional factors and brain alterations were retrieved from the eligible papers. Examples of questionnaires providing information on self-reported maladaptive cognitive/emotional factors related to pain are, among others, the 
Pain Catastrophizing Scale (PCS), the Tampa Scale for Kinesiophobia (TSK) and, the Hospital Anxiety and Depression Scale (HADS). Examples of MRI techniques providing information on brain alterations are, among others, Voxel-Based Morphometry (VBM), Blood Oxygenation Level-Dependent (BOLD) functional MRI and, Diffusion Tensor Imaging (DTI). A level of conclusion was determined after clustering studies with comparable methods or results, accounting for the study designs and the risk of bias ("Supporting Information Table S3").

\section{Results}

\subsection{Study selection}

The selection process of the relevant papers can be found in Fig. 1. The initial search resulted in a total of 1056 hits, of which 781 papers remained after deduplication. After screening each manuscript on title and abstract for in- and exclusion criteria, 83

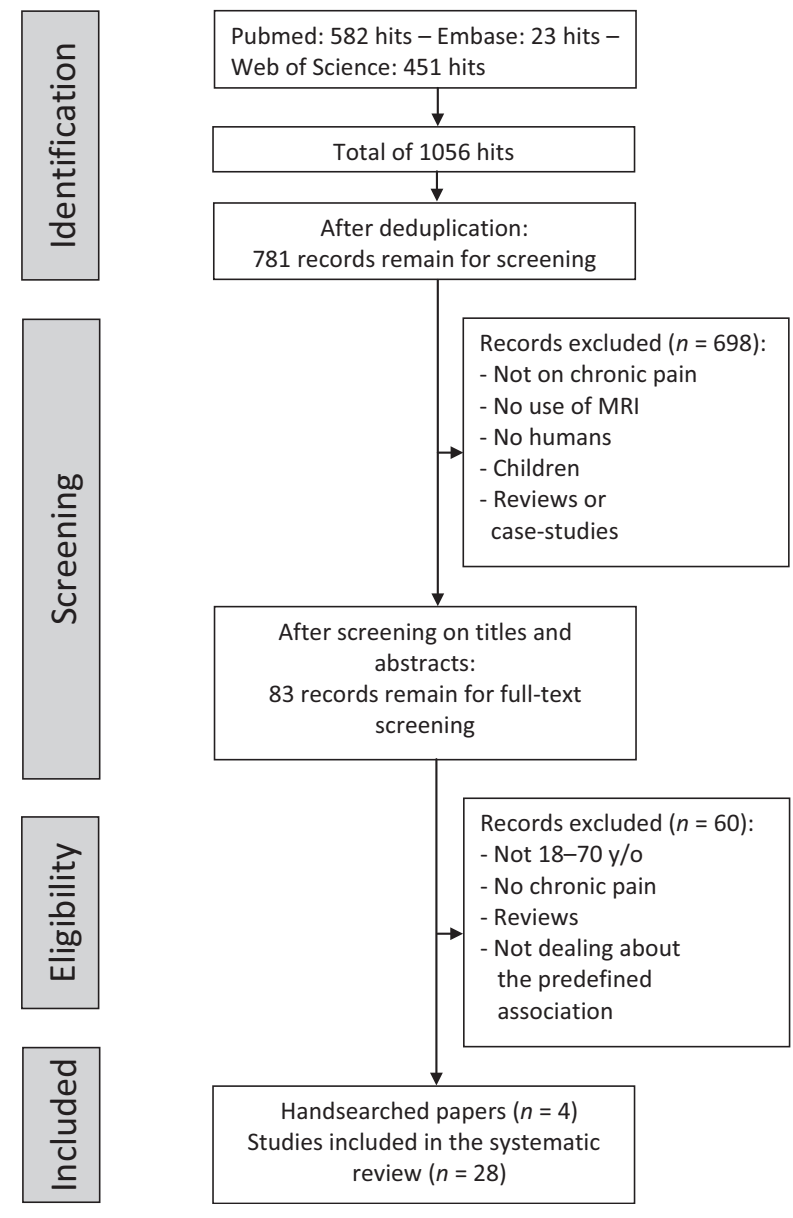

Figure 1 Flowchart: Selection process. studies remained for screening the full text. Reference lists of relevant papers were screened for suitable papers, which resulted in four eligible papers that were not found by the electronic search strategy. Finally, this screening process led to the inclusion of 28 suitable articles.

\subsection{Study characteristics}

Twenty eight studies were found eligible for this systematic review, of which the majority (24 of 27) were case-control studies (Schmidt-Wilcke et al., 2007; Geha et al., 2008; Lutz et al., 2008; Hsu et al., 2009; Valet et al., 2009; Blankstein et al., 2010; Elsenbruch et al., 2010; Burgmer et al., 2011; Gerstner et al., 2011; Glass et al., 2011; Gustin et al., 2011; As-Sanie et al., 2012; Barke et al., 2012; Cifre et al., 2012; Ceko et al., 2013, 2015; Hong et al., 2013; Kim et al., 2013; Seminowicz et al., 2013; Khan et al., 2014; Kucyi et al., 2014; Lloyd et al., 2014; Gupta et al., 2015; Riederer et al., 2015). Three studies were described as observational (cross-sectional; Loggia et al., 2015; Lloyd et al., 2008; Gracely et al., 2004) and only one study was a RCT (RCT; Jensen et al., 2012). Ten studies included men and women (Gracely et al., 2004; Schmidt-Wilcke et al., 2007; Geha et al., 2008; Lloyd et al., 2008, 2014; Gustin et al., 2011; Cifre et al., 2012; Hong et al., 2013; Loggia et al., 2015; Riederer et al., 2015), while the others only included women. The mean age range over all included studies was quite large, going from $24.4 \pm 1.5$ to $54 \pm 8$ years old. Detailed information on these study characteristics is presented in Table 2.

A variety of MRI techniques were used in the included studies, and information was found on changes in both brain structure and function associated with maladaptive cognitive/emotional factors related to pain, being pain catastrophizing, fearavoidance, anxiety and depressive symptoms.

\subsection{Risk of bias and level of evidence}

A $93.8 \%$ agreement on scores of methodological quality was achieved between the two researchers. Disagreement occurred mainly due to differences in interpretation of criteria, but a consensus was reached without having to consult the third researcher. Detailed information on the methodological quality and level of evidence of the included studies is listed in Table 3.

Overall, global rating was strong in five studies (Valet et al., 2009; Glass et al., 2011; As-Sanie et al., 


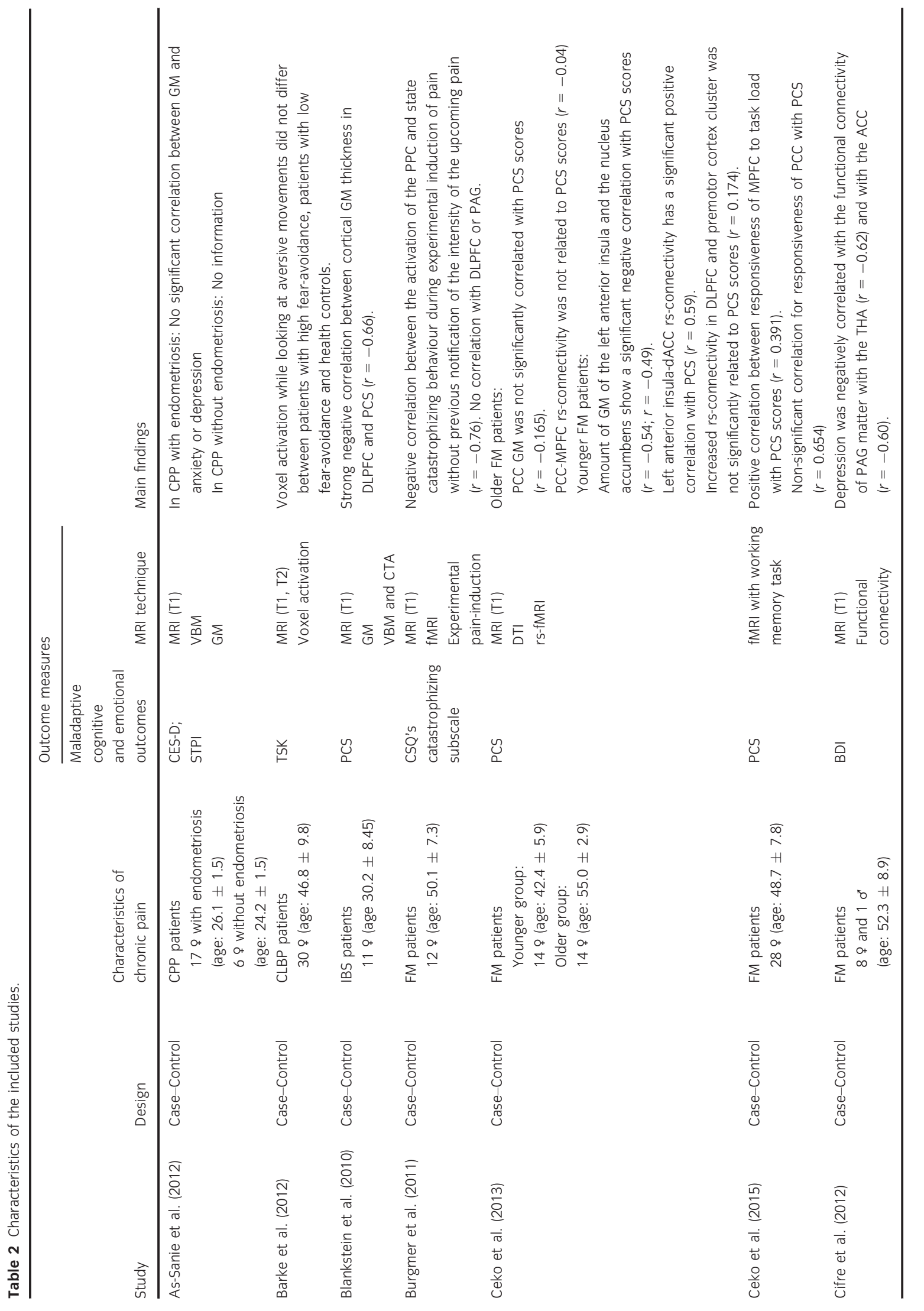




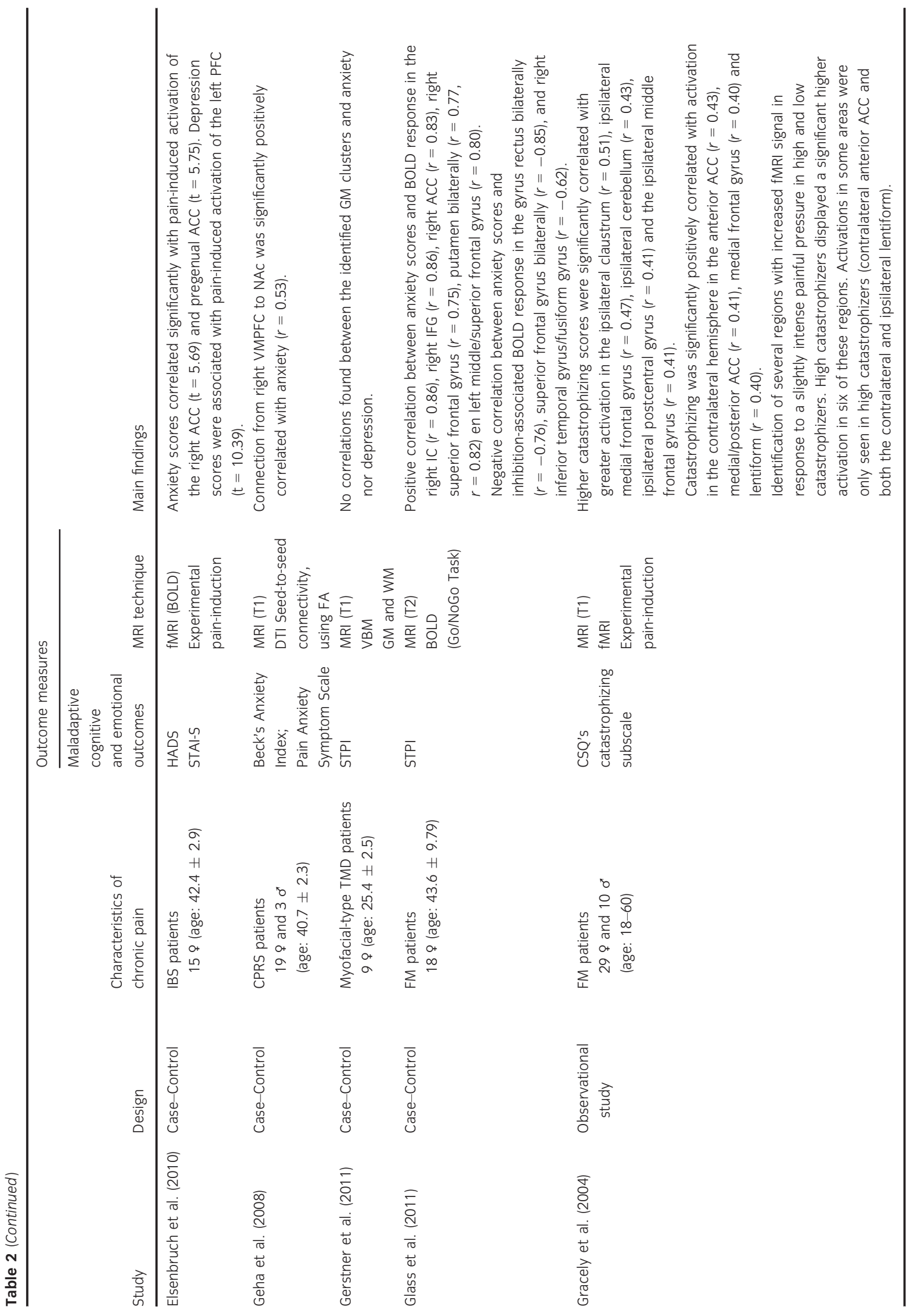




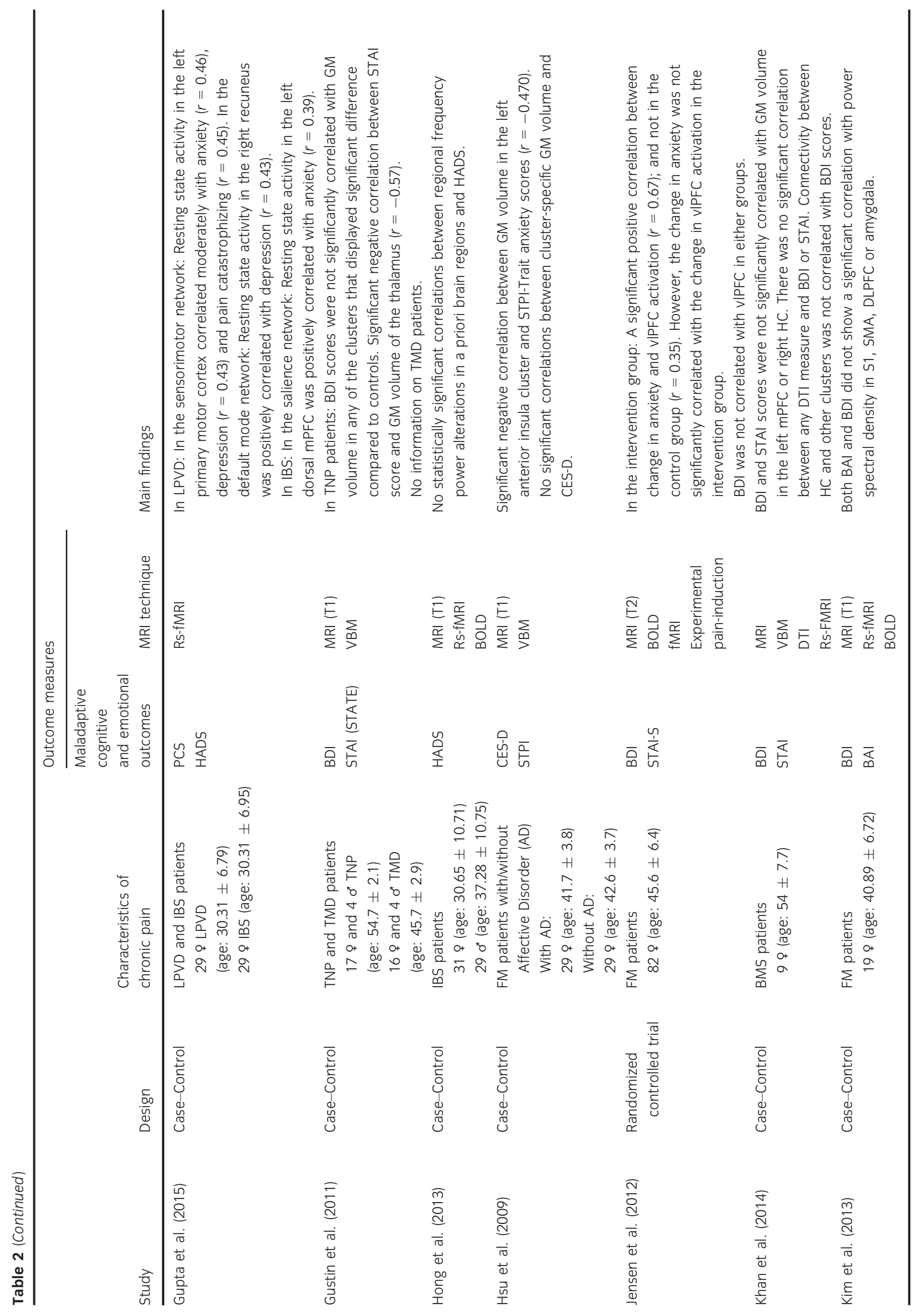




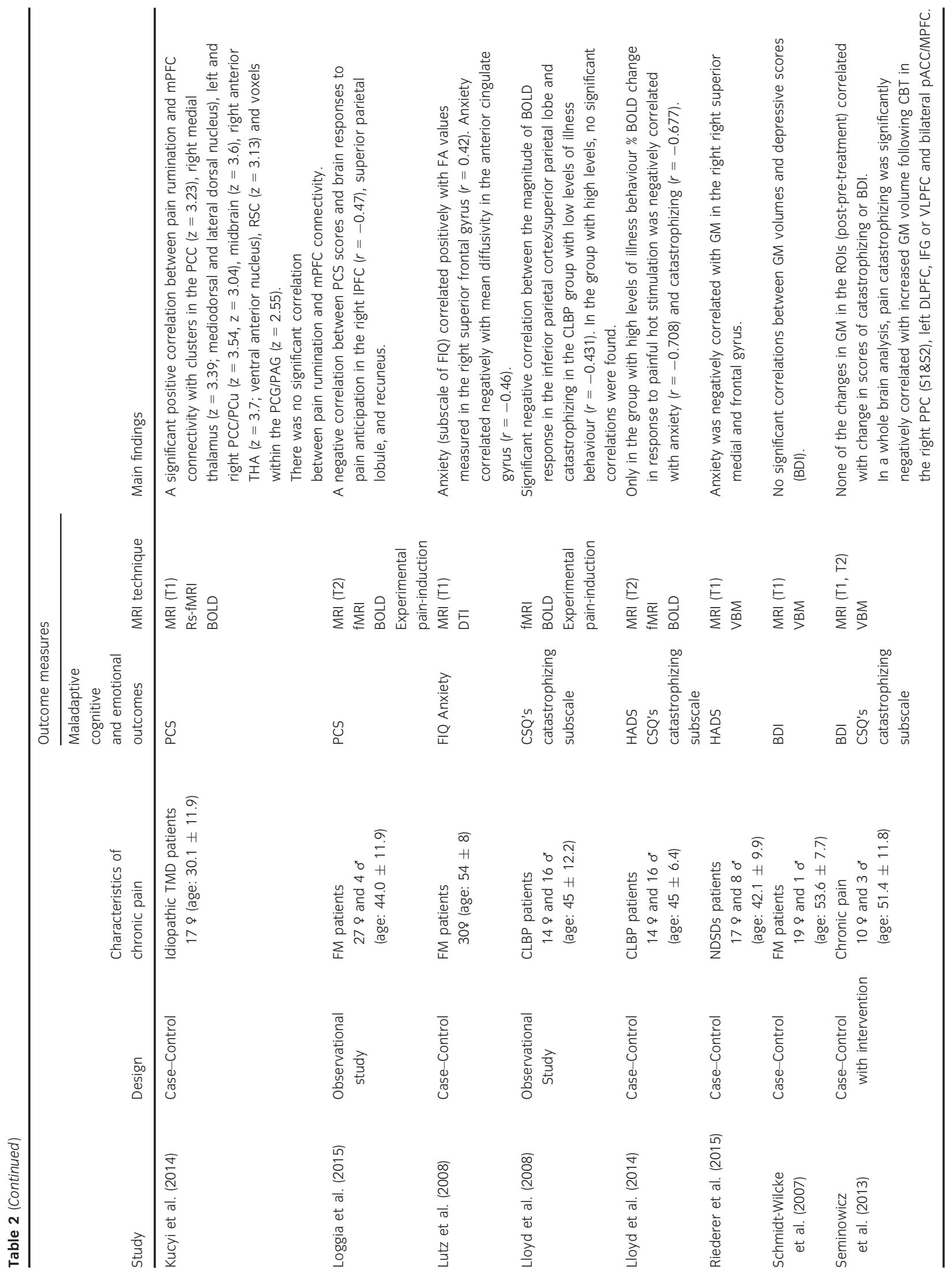




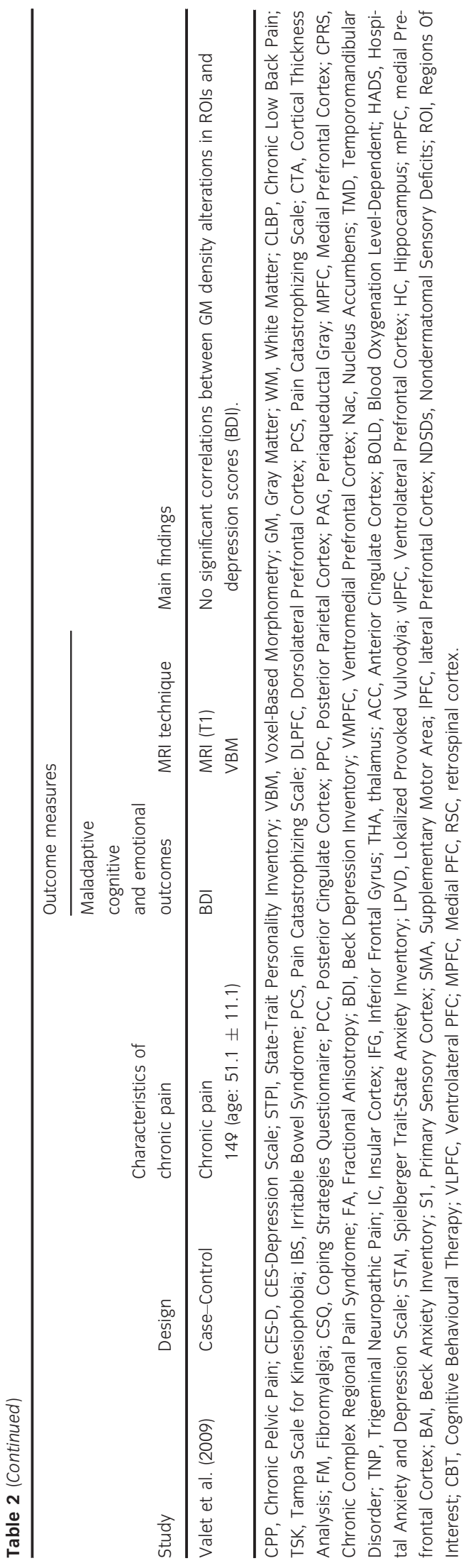

2012; Jensen et al., 2012; Gupta et al., 2015), moderate in 12 studies (Schmidt-Wilcke et al., 2007; Geha et al., 2008; Lloyd et al., 2008; Hsu et al., 2009; Blankstein et al., 2010; Gustin et al., 2011; Barke et al., 2012; Ceko et al., 2013, 2015; Hong et al., 2013; Kim et al., 2013; Riederer et al., 2015) and weak in 11 studies (Gracely et al., 2004; Lutz et al., 2008; Elsenbruch et al., 2010; Burgmer et al., 2011; Gerstner et al., 2011; Cifre et al., 2012; Seminowicz et al., 2013; Khan et al., 2014; Kucyi et al., 2014; Lloyd et al., 2014; Loggia et al., 2015). Thirteen of 27 studies scored weak on selection bias (criterion A), mostly due to a lack of adequate description of subject selection procedure. The strength of the study design was deemed moderate in all but one study, which was rated strong for being a RCT (criterion B). Twelve studies were rated strong for the description of confounders (criterion C), five were found to be moderate and all others obtained a weak score as they did not account appropriately for possible confounders. In all but one study, data collection methods were strong. In these studies, used questionnaires and MRI techniques were standard tools with known reliability and validity. The one study that scored moderate used a modified version of a questionnaire which they described as not validated as such (Burgmer et al., 2011). Withdrawals and drop-outs (criterion F) were clearly described in eleven studies (strong), moderately in four studies and essentially missing (weak) in 13 studies. Finally, MRI data quality and pre-processing (criterion G) was guaranteed in most studies as only one study scored weak on this criterion (Burgmer et al., 2011).

\subsection{Association between maladaptive cognitive or emotional factors and brain alterations in chronic pain}

Results will be grouped based on maladaptive cognitive or emotional factors and will be given a level of conclusion (Table S3) per cluster of results.

\subsubsection{Fear-avoidance}

Only one study investigated brain aspects in relation to fear-avoidance. Barke et al. (2012) studied the neural correlates of high and low fear-avoidant chronic low back pain patients. Voxel activation while looking at aversive movements did not differ between patients with high fear-avoidance, patients with low fear-avoidance and healthy controls, meaning that fear-avoidance was not associated with a 
Table 3 Methodological quality of included studies.

\begin{tabular}{|c|c|c|c|c|c|c|c|c|c|}
\hline \multirow[b]{2}{*}{ Study } & \multicolumn{7}{|c|}{ Component ratings } & \multirow[b]{2}{*}{ Global rating } & \multirow[b]{2}{*}{ LOE } \\
\hline & A & B & $\mathrm{C}$ & D & $\mathrm{E}$ & $\mathrm{F}$ & G & & \\
\hline As-Sanie et al. (2012) & Moderate & Moderate & Strong & Moderate & Strong & Strong & Strong & Strong & B \\
\hline Barke et al. (2012) & Moderate & Moderate & Weak & Moderate & Strong & Strong & Moderate & Moderate & B \\
\hline Blankstein et al. (2010) & Moderate & Moderate & Weak & Moderate & Strong & Moderate & Strong & Moderate & B \\
\hline Burgmer et al. (2011) & Moderate & Moderate & Weak & Moderate & Moderate & Strong & Weak & Weak & B \\
\hline Ceko et al. (2013) & Weak & Moderate & Strong & Moderate & Strong & Strong & Strong & Moderate & B \\
\hline Ceko et al. (2015) & Weak & Moderate & Strong & Moderate & Strong & Strong & Strong & Moderate & B \\
\hline Cifre et al., 2012; & Weak & Moderate & Weak & Moderate & Strong & Weak & Moderate & Weak & B \\
\hline Elsenbruch et al. (2010) & Moderate & Moderate & Weak & Moderate & Strong & Weak & Moderate & Weak & B \\
\hline Geha et al. (2008) & Moderate & Moderate & Weak & Moderate & Strong & Moderate & Moderate & Moderate & B \\
\hline Gerstner et al. (2011) & Weak & Moderate & Strong & Moderate & Strong & Weak & Strong & Weak & B \\
\hline Glass et al. (2011) & Moderate & Moderate & Strong & Moderate & Strong & Strong & Strong & Strong & B \\
\hline Gracely et al. (2004) & Weak & Moderate & $\mathrm{N} / \mathrm{A}$ & Moderate & Strong & Weak & Strong & Weak & B \\
\hline Gupta et al. (2015) & Moderate & Moderate & Strong & Moderate & Strong & Strong & Strong & Strong & B \\
\hline Gustin et al. (2011) & Weak & Moderate & Strong & Moderate & Strong & Moderate & Moderate & Moderate & B \\
\hline Hong et al. (2013) & Moderate & Moderate & Moderate & Moderate & Strong & Weak & Moderate & Moderate & B \\
\hline Hsu et al. (2009) & Weak & Moderate & Strong & Moderate & Strong & Strong & Strong & Moderate & B \\
\hline Jensen et al. (2012) & Moderate & Strong & Strong & Moderate & Strong & Moderate & Strong & Strong & $\mathrm{A} 2$ \\
\hline Khan et al. (2014) & Moderate & Moderate & Weak & Moderate & Strong & Weak & Moderate & Weak & B \\
\hline Kim et al. (2013) & Moderate & Moderate & Moderate & Moderate & Strong & Weak & Moderate & Moderate & B \\
\hline Kucyi et al. (2014) & Weak & Moderate & Moderate & Moderate & Strong & Weak & Strong & Weak & B \\
\hline Lloyd et al. (2008) & Weak & Moderate & Strong & Moderate & Strong & Strong & Moderate & Moderate & B \\
\hline Lloyd et al. (2014) & Weak & Moderate & Weak & Moderate & Strong & Strong & Moderate & Weak & B \\
\hline Loggia et al. (2015) & Weak & Moderate & $\mathrm{N} / \mathrm{A}$ & Moderate & Strong & Weak & Strong & Weak & B \\
\hline Lutz et al. (2008) & Weak & Moderate & Strong & Moderate & Strong & Weak & Strong & Weak & B \\
\hline Riederer et al. (2015) & Moderate & Moderate & Strong & Moderate & Strong & Weak & Moderate & Moderate & B \\
\hline Schmidt-Wilcke et al. (2007) & Moderate & Moderate & Moderate & Moderate & Strong & Weak & Strong & Moderate & B \\
\hline Seminowicz et al. (2013) & Weak & Moderate & Weak & Moderate & Strong & Weak & Strong & Weak & B \\
\hline Valet et al., 2009 & Moderate & Moderate & Moderate & Moderate & Strong & Strong & Strong & Strong & B \\
\hline
\end{tabular}

N/A, Not Applicable; LOE, Level of Evidence.

Components of the EPHPP Quality Assessment Tool: A: Selection Bias (Are the individuals selected to participate in the study likely to be representative of the target population? AND What percentage of selected individuals agreed to participate?); B: Study Design (Indicate the study design. AND Was the study described as randomized? If yes, was the method of randomization described? If yes, was the method appropriate?); C: Confounders (Were there important differences between groups prior to the intervention? AND If yes, indicate the percentage of relevant confounder that were controlled.); D: Blinding (Were the outcome assessors aware of the intervention or status of the participants? AND Were the study participants blind or aware of the research question?); E: Data Collection Methods (Were data collection tools shown to be valid? AND Were data collection tools shown to be reliable?); F: Withdrawals and drop-outs (Were withdrawals and drop-outs reported in terms of numbers and/or reasons per group? AND Indicate the percentage of participants completing the study.); G: MRI data quality and pre-processing (Was visual inspection of MRI data quality performed? AND Did the researchers apply manual exclusion in case of low data quality and/or was data adjustment included in the pre-processing pipeline?).

different level of voxel activation (Strength of Conclusion 3). Results of this study should be interpreted in the light of the weak methodological quality score this study received.

\subsubsection{Pain catastrophizing}

Nine studies looked at the association between pain catastrophizing and gray matter morphology, resting state functional connectivity, and task-related functional brain activation (Gracely et al., 2004; Lloyd et al., 2008, 2014; Blankstein et al., 2010; Burgmer et al., 2011; Seminowicz et al., 2013; Kucyi et al., 2014; Gupta et al., 2015; Loggia et al., 2015).
3.4.2.1 Gray matter morphology. Three studies investigated the association between pain catastrophizing and gray matter morphology (Blankstein et al., 2010; Ceko et al., 2013; Seminowicz et al., 2013). Blankstein et al. (2010) found that higher pain catastrophizing in patients with irritable bowel syndrome is associated with thinning of gray matter in the Dorsolateral Prefrontal Cortex (DLPFC; strong negative correlation when accounted for age). Seminowicz et al. (2013) confirm the presence of this association in people with chronic pain. They report that a decrease in pain catastrophizing following cognitive 
behavioural therapy was significantly correlated with a gray matter increase in the left DLPFC, as well as a gray matter increase in the right Posterior Parietal Cortex that included parts of the primary (S1) and secondary (S2) somatosensory cortex, the Inferior Frontal Gyrus or Ventrolateral Prefrontal Cluster, and the bilateral Anterior Cingulate Cortex/Medial Prefrontal Cortex (mPFC). However, the right Hippocampus and the right DLPFC were significantly positively correlated with pain catastrophizing. Ceko et al. (2013) on the other hand found a significant negative correlation between pain catastrophizing and the amount of gray matter of the nucleus accumbens and the left anterior insula in the younger group of fibromyalgia patients, while they did not find a significant correlation with the Posterior Cingulate Cortex (PCC) gray matter in the older patients.

To conclude, there is moderate evidence for an association between pain catastrophizing and alterations in gray matter morphology in the DLPFC (strength of conclusion 2), but evidence is inconclusive regarding the direction of this relation. Additionally, there is some evidence for a negative association of pain catastrophizing and gray matter volume in brain areas involved in somatosensory, motor and pain processing (strength of conclusion 3).

3.4.2.2 Resting state functional activity. Three other studies investigated the relation between pain catastrophizing and resting state functional activity (Ceko et al., 2013; Kucyi et al., 2014; Gupta et al., 2015). Kucyi et al. (2014) studied patients with chronic temporomandibular disorders and found a significant positive correlation between pain rumination and the mPFC functional connectivity with clusters including peak voxels in the left/right Posterior Cingulate Cortex/Precuneus, the medial and anterior thalamus, the Retrosplenial Cortex, and voxels within the Periventricular Gray/ Periaquaductal Gray. They also suggest that individuals who display higher levels of pain rumination most strongly drive enhanced mPFCPosterior Cingulate Cortex/Precuneus functional connectivity. Gupta et al. (2015) on the other hand, investigated this association in both patients with irritable bowel syndrome and with localized provoked vulvodynia and found that for the sensorimotor network pain catastrophizing correlated positively with the left primary motor cortex when patients with vulvodynia were compared to patients with irritable bowel syndrome. Last, Ceko et al.
(2013) included fibromyalgia patients and found a significant positive correlation between pain catastrophizing and the left anterior insula and dorsal Anterior cingulate cortex (ACC) resting state connectivity in the younger subgroup. They also investigated the relation between pain catastrophizing and resting state connectivity of both PCC-MPFC and DLPFC-right premotor cortex but found no significant correlations.

To conclude, there is some evidence for a positive association between pain catastrophizing and resting state functional connectivity in brain areas involved in pain, sensorimotor and affective-cognitive processing in patients with chronic temporomandibular disorders, vulvodynia and fibromyalgia (strength of conclusion 3).

3.4.2.3 Task related brain activation. Regarding taskrelated brain activation, four studies investigated the relation between pain catastrophizing and experimental pain-induced brain activation in chronic pain (Gracely et al., 2004; Lloyd et al., 2008, 2014; Burgmer et al., 2011), one study investigated this relation in response to pain anticipation (Loggia et al., 2015) and one study applied a working memory task (Ceko et al., 2015).

The two studies of Lloyd et al. (2008, 2014) focussed in particular on patients with chronic low back pain. In their first study (Lloyd et al., 2008) they found a significant negative correlation between pain catastrophizing and the magnitude of BOLD response after intense tactile stimulation in the midline retrosplenial cingulate cortex, right striate and extrastriate cortex, and left inferior and superior parietal cortex. However, in their second study (Lloyd et al., 2014), when using a heat noxious stimulus, they found a similar significant negative correlation that did not survive correction for multiple comparison.

The two other studies focusing on pain-induced brain activation investigated fibromyalgia patients. Gracely et al. (2004) found that in response to slightly intense pressure pain, pain catastrophizing was significantly and positively associated with greater activation in the ipsilateral claustrum, the ipsilateral medial frontal gyrus, the ipsilateral cerebellum, the ipsilateral postcentral gyrus, the ipsilateral middle frontal gyrus, in the contralateral anterior/medial/posterior anterior cingulate cortex, the contralateral medial frontal gyrus and the contralateral lentiform. In the study of Burgmer et al. (2011) a significant correlation was found between pain catastrophizing and the activation of the 
posterior parietal cortex during induction of pain without prior notice of the intensity of the upcoming pain.

One study investigated the relation between pain catastrophizing and brain activation in response to pain anticipation (Loggia et al., 2015) and found a negative correlation between pain catastrophizing scores and activation of the right lateral prefrontal cortex, the superior parietal lobule and the precuneus.

The study including a working memory task in fibromyalgia patients found a significant positive correlation between pain catastrophizing and the responsiveness of the $\mathrm{mPFC}$ to the task load, while they did not find a significant correlation with the responsiveness of the PCC (Ceko et al., 2015).

To conclude, there is some evidence for an association between pain catastrophizing and activation in response to experimentally induced pain in brain areas involved in pain, motor, somatosensory and affective-cognitive processing. Although results are inconclusive, evidence is in favour of a negative association (strength of conclusion 3). As evidence is limited to one study, no conclusions can be drawn on brain activation in response to pain anticipation or a working memory task.

\subsubsection{Anxiety}

A lot of research (14 studies) has been conducted to understand the association of anxiety with gray matter morphology, white matter properties, resting state functional activity and task-related activation of the brain in chronic pain (Geha et al., 2008; Lutz et al., 2008; Hsu et al., 2009; Elsenbruch et al., 2010; Gerstner et al., 2011; Glass et al., 2011; As-Sanie et al., 2012; Jensen et al., 2012; Hong et al., 2013; Kim et al., 2013; Khan et al., 2014; Lloyd et al., 2014; Gupta et al., 2015; Riederer et al., 2015).

3.4.3.1 Gray matter morphology. Seven studies investigated the association between anxiety and gray matter morphology alterations in chronic pain patients (Lutz et al., 2008; Hsu et al., 2009; Gerstner et al., 2011; Gustin et al., 2011; As-Sanie et al., 2012; Khan et al., 2014; Riederer et al., 2015). Three of them found a significant negative correlation between anxiety and gray matter volume: in the right superior medial and frontal gyrus in chronic pain patients with nondermatomal somatosensory deficits (Riederer et al., 2015), in the left anterior insula in fibromyalgia patients (Hsu et al., 2009) and in the thalamus in patients with trigeminal neuropathic pain (Gustin et al., 2011). The four studies that did not find a significant correlation were investigating patients with temporomandibular disorders (Gerstner et al., 2011), chronic pelvic pain (As-Sanie et al., 2012), chronic burning mouth syndrome (Khan et al., 2014) and fibromyalgia (Lutz et al., 2008).

In addition to these six studies, the study of Geha et al. (2008) performed seed to seed connectivity analyses between gray matter regions and found that the connection count from the right ventromedial prefrontal cortex to the nucleus accumbens was significantly positively correlated with two separate anxiety scores in CPRS patients.

To conclude, evidence on the association between self-reported anxiety and gray matter morphology alterations is ambiguous. While the majority of studies lacks an association, there is some evidence for an association of anxiety with gray matter alterations in affective-cognitive brain areas, however, evidence is inconclusive regarding the direction of this association (strength of conclusion 3).

3.4.3.2 White matter properties. Two studies investigated the association between anxiety and white matter properties (Lutz et al., 2008; Khan et al., 2014). One study included patients with chronic burning mouth syndrome and did not find any significant correlation (Khan et al., 2014). The study investigating patients with fibromyalgia found both a positive significant correlation between anxiety and fractional anisotropy in the right superior frontal gyrus and a significant negative correlation between anxiety and mean diffusivity (apparent diffusion coefficient) in the ipsilateral anterior cingulate cortex (Lutz et al., 2008). Fractional anisotropy is a measure of the degree of diffusion anisotropy.

To conclude, there is limited evidence for a positive association of fractional anisotropy in affectivecognitive brain areas and a negative association of mean diffusivity in pain processing brain areas with self-reported anxiety (strength of conclusion 3).

3.4.3.3 Resting state functional activity. Three studies investigated the association between resting state functional activity and anxiety (Hong et al., 2013; Kim et al., 2013; Gupta et al., 2015). Two studies did not find any significant correlation of anxiety with a priori selected brain regions (DLPFC, amygdala, primary somatosensory cortex or supplementary motor area, insula and hippocampus; Kim et al., 2013; Hong et al., 2013), while the third 
study found a significant positive correlation in the salience network between anxiety and the left dorsal medial prefrontal cortex in irritable bowel syndrome patients compared to patients with localized provoked vulvodynia (Gupta et al., 2015).

To conclude, there is limited evidence for a positive correlation of anxiety with the salience network in affective-cognitive brain areas in patients with irritable bowel syndrome (strength of conclusion 3).

3.4.3.4 Task-related brain activation. Four studies looked at task-related brain activation in relation to anxiety, of which three used experimental pain-induction (Elsenbruch et al., 2010; Jensen et al., 2012; Lloyd et al., 2014) and one used a Go/No Go Task (patients had to respond to target letters by pressing a button; Glass et al., 2011).

Experimental pain-induction was used by Lloyd et al. (2014), who investigated chronic low back pain patients and found a significant negative correlation between anxiety and BOLD signal change in several brain areas, but none of the changes survived the correction for multiple comparison. Jensen et al. (2012) studied experimental pain-induced brain activation in fibromyalgia patients, but focussed at the changes after cognitive behavioural therapy and found that the therapy-related change in anxiety was positively correlated with ventrolateral prefrontal cortex activation, but this significance disappeared after further analyses. Last, Elsenbruch et al. (2010) studied experimental pain-induced brain activation in patients with irritable bowel syndrome and found a significant positive correlation between anxiety and activation in the right anterior midcingulate cortex and the pregenual ACC.

Glass et al. (2011) on the other hand, studied fibromyalgia patients during an attention task (response inhibition) and found that anxiety scores correlated positively with BOLD response in the right insular cortex, right inferior and superior frontal gyrus, right anterior cingulate cortex, putamen bilaterally, and left middle/superior temporal gyrus.

To conclude, evidence mostly lacks an association, but there is some evidence for a positive association between anxiety and task-related brain activation in affective-cognitive and pain processing areas (strength of conclusion 3).

\subsubsection{Depressive symptoms}

Last, 14 articles provide information regarding the relation between depressive symptoms measured by self-reported questionnaires and brain alterations, including gray matter morphology, white matter properties, resting state functional activity and taskrelated brain activation (Schmidt-Wilcke et al., 2007; Lutz et al., 2008; Hsu et al., 2009; Valet et al., 2009; Elsenbruch et al., 2010; Gerstner et al., 2011; AsSanie et al., 2012; Cifre et al., 2012; Jensen et al., 2012; Hong et al., 2013; Kim et al., 2013; Seminowicz et al., 2013; Khan et al., 2014; Gupta et al., 2015).

3.4.4.1 Gray matter morphology and white matter properties. Eight studies investigated the relation between gray matter (volume or density) changes and depressive symptoms, but none of them found a significant association (Schmidt-Wilcke et al., 2007; Hsu et al., 2009; Valet et al., 2009; Gerstner et al., 2011; Gustin et al., 2011; As-Sanie et al., 2012; Seminowicz et al., 2013; Khan et al., 2014). Likewise, with regard to white matter properties no association was shown with depressive symptoms (Lutz et al., 2008; Khan et al., 2014).

To conclude, when looking at self-reported depressive symptoms, no evidence was found for an association with alterations in gray matter morphology and white matter properties (strength of conclusion $3)$.

3.4.4.2 Resting state functional activity. Two studies did not find any significant association of depressive symptoms with resting-state functional activity in a priori selected brain regions (DLPFC, amygdala, primary somatosensory cortex or supplementary motor area, insula and hippocampus) in fibromyalgia and irritable bowel syndrome (Hong et al., 2013; Kim et al., 2013). However, a significant positive correlation was found between depressive symptoms and the default mode network in the right precuneus in localized provoked vulvodynia (Gupta et al., 2015). On the other hand, Cifre et al. (2012) demonstrated a significant negative correlation between depressive symptoms and resting state functional connectivity of periaqueductal gray matter with the thalamus and with the anterior cingulate cortex in fibromyalgia patients.

To conclude, although there are two studies not finding any significant associations, there is some evidence for a negative association between selfreported depressive symptoms and resting state functional connectivity in brain areas involved in pain processing, while there is also some evidence for a negative association with parts of the default mode 
network involved in affective-cognitive processing (strength of conclusion 3).

3.4.4.3 Task-related brain activation. Two studies looked at task-related brain activation in response to experimental pain-induction in relation to depressive symptoms. Jensen et al. (2012) focussed on the ventrolateral prefrontal cortex but did not find a significant correlation, while Elsenbruch et al. (2010) found a significant positive correlation with activation in the left prefrontal cortex.

\section{Discussion}

This review aimed at providing an overview of existing knowledge on the relation between brain alterations and maladaptive cognitive and emotional factors in patients with chronic pain. The search of relevant literature has led to the inclusion of 28 articles, providing limited to moderate evidence that brain alterations are indeed associated with certain self-reported cognitive and emotional factors in patients with chronic pain.

From the present systematic literature study it can be concluded that there is some evidence for an association of pain catastrophizing with alterations in gray matter morphology, resting state functional connectivity and task-related brain activation in brain areas involved in pain, motor, somatosensory and affective-cognitive processing. It can be concluded that the evidence of an association between anxiety and alterations in gray matter morphology is ambiguous, and that limited evidence is found regarding the association with resting state functional activity and task-related brain activation in affective-cognitive brain areas. For self-reported depressive symptoms this review concludes no evidence for an association with gray and white matter properties, while evidence regarding the association with resting state functional connectivity and taskrelated brain activation is inconclusive with studies reporting both an absence of a significant association as well as limited evidence for associations with pain processing and affective-cognitive brain areas. This is important because having insight on the relation of brain alterations with maladaptive pain related cognitive and emotional factors will lead to more understanding of the underlying and sustaining mechanisms of chronic pain.

Indeed, the challenge of chronic pain lies in its multidimensional character, being influenced by psychosocial factors such as, previous experiences, cultural beliefs, pain cognitions, emotional factors and coping responses, in addition to biological factors like sensory input (Melzack and Wall, 1982; Jensen et al., 2002). When looking at cognitive and emotional factors, literature shows abundant evidence for their role in the chronification of pain (Bigos et al., 1992; Burton et al., 1995; Apkarian et al., 2005; Wiech et al., 2008; Wiech and Tracey, 2009) and it is known that pain duration is associated with the extent of measured brain alterations in patients with chronic pain. To date, the underlying mechanisms of the established brain alterations in the chronic pain population remain unclear, but as evidence provides an explanatory framework for pain chronification with both brain alterations and maladaptive pain cognitions and emotions, the mutual association between these elements becomes very relevant. The presence of such associations provides a clinical relevance to these reported brain changes as they could lead to the hypothesis that a specific treatment program could influence or even reverse these brain changes by addressing cognitions and emotions.

A key finding of this systematic review is that there is clear evidence that brain alterations, including gray matter volume, functional connectivity in the resting state network and task-related brain activation, are undeniably associated with self-reported pain catastrophizing (Gracely et al., 2004; Lloyd et al., 2008, 2014; Blankstein et al., 2010; Burgmer et al., 2011; Seminowicz et al., 2013; Kucyi et al., 2014; Loggia et al., 2015). Pain catastrophizing is defined as having difficulties with shifting focus or attention away from painful or threatening stimuli, and denoting more threat or harm to non-painful stimuli (Crombez et al., 1998). The reported significant associations indicate that catastrophizing is related to pain processing (Loggia et al., 2015), is associated with reduced engagement of the descending pain modulatory system (Blankstein et al., 2010; Kucyi et al., 2014; Loggia et al., 2015) and is associated with activation of brain areas involved in attention to pain, emotion and motor activity (Gracely et al., 2004).

Although the direction of the associations between pain catastrophizing and brain characteristics is ambiguous, a certain trend can be detected with several positive associations regarding brain activations and more prevalent negative associations regarding gray matter properties. This could indicate that chronic pain patients who tend to catastrophize more show indeed stronger brain activations, meaning that in these patients the brain is working 'more' in response to pain- 
induction or even spontaneous pain as described in previous research (Baliki et al., 2006). On the other hand higher catastrophizing seems to be related to decreased gray matter. For the prefrontal regions this could be explained by the fact that these regions are responsible for the topdown inhibition of orbitofrontal activity and thus are able to influence the level of perceived pain. Atrophy of gray matter in these regions could indicate difficulties in the control of the orbitofrontal region and thus lead to more negative perceptions and catastrophizing in general (Apkarian et al., 2004).

Another key finding of this review is the fact that all identified pain-related cognitive and emotional factors are indeed showing significant associations with the data obtained during the resting state fMRI scans. The resting state scan is used to evaluate regional brain interactions that occur when the scanned subject is not engaged in an explicit task. This specific scan can give information regarding the default mode network, which is defined as a set of synchronous brain regions that are active at rest and are deactivated during performance of a task and is said to be the first network affected by chronic pain (Baliki et al., 2014). The results presented in this review provide evidence that pain catastrophizing, anxiety and depressive symptoms seem to be involved in altering this network. Therefore we can hypothesize that they can serve as a 'mind wandering-like' distraction away from a certain cognitive task (Kucyi et al., 2014; Ceko et al., 2015), meaning that even in a 'mind at rest' a certain share of brain activity in affective-cognitive areas is reserved for pain-related catastrophizing, anxiety and depressive symptoms.

This review reports many findings, but as they are limited to correlations, it remains difficult to formulate a specific hypothesis on the causality of these associations. Nevertheless, two studies report on the use of cognitive behavioural therapy and its effect on brain alterations related to both pain catastrophizing (Seminowicz et al., 2013) and anxiety (Jensen et al., 2012). The results of these studies indicate that cognitive behavioural therapy can decrease both pain catastrophizing and anxiety, leading to positive effects on gray matter volume and brain activation in prefrontal brain regions related to executive function, emotions and coordination of selective attention. This leads to the hypothesis that when pain catastrophizing and anxiety decrease, less attention is given to pain and pain is less accompanied by negative emotions, which is reflected in a positive change in brain alterations.

\section{Limitations}

It has to be mentioned that some authors report technical issues (Geha et al., 2008; Riederer et al., 2015) or small study populations (Burgmer et al., 2011; Gerstner et al., 2011; As-Sanie et al., 2012), both possibly influencing the reported results. It has to be acknowledged that the used magnetic field strength, voxel size and MRI processing techniques varied across the included studies, making results difficult to interpret. Nevertheless, taking these elements into account, this review aimed at providing a broad oversight of existing literature on this subject, while trying to refrain from drawing too specific conclusions.

Additionally, it is important to take gender differences in brain function and morphology into account. Some studies reported results of both men and women together, while other studies only included women and not all studies took medication or hormonal status (in case of females) into consideration. This is a limitation, as gender differences in brain characteristics are acknowledged in literature (Alarcon et al., 2015; Scheinost et al., 2015; Xu et al., 2015). The same applies for the large range of mean age of the subjects over the included articles as age also has a great impact on brain characteristics (Hogstrom et al., 2013; Alarcon et al., 2015; Scheinost et al., 2015).

Another limitation comprises the use of pearson and spearman correlations in most studies to investigate the relation between brain alterations and maladaptive cognitive and emotional factors. It is wellknown that these type of correlations are limited for interpretation and that linear regression techniques are preferred to investigate these type of relations, which was only used in some of the included studies (Schmidt-Wilcke et al., 2007; Glass et al., 2011; Loggia et al., 2015; Riederer et al., 2015).

Furthermore, it has to be acknowledged that the chronic pain population covers heterogeneous pain syndromes. This might deliver an explanation for the inconclusive results in this systematic review, as every chronic pain condition appears to have its specific characteristic brain changes, leading to an unique anatomical 'brain signature' (Baliki et al., 2011). Nevertheless, evidence has shown that many chronic pain conditions share mutual characteristic brain changes, with brain alterations in key regions like the DLPFC, thalamus, brainstem and somatosensory cortex (Apkarian et al., 2004; Buckalew et al., 2008; 
Seminowicz et al., 2011). Moreover, many chronic pain conditions are not represented in this systematic review due to lack of research. For example, nothing can be found on the relationship of brain alterations and pain cognitive and emotional factors in patients with chronic neck pain (traumatic nor non-traumatic), chronic osteoarthritis pain, chronic shoulder pain, etc. Also, unfortunately a major part of the included studies explored the research question of this systematic review as a secondary objective, leading to limited information.

Finally, it should be noted that because of the study designs of the included studies, no conclusions can be drawn on the causality of the observed associations. It is unclear whether the observed brain changes are the cause or the consequence of maladaptive pain cognitive and emotional factors, and by extend of chronic pain.

\section{Recommendations for further research}

As this review reveals, literature on cognitive and emotional factors related to brain alterations in chronic pain patients is limited to fear-avoidance, pain catastrophizing, anxiety and depressive symptoms. Information on pain and illness perceptions, pain attention, beliefs, attitudes, etc. is currently lacking, although these factors have an established contribution to the occurrence and persistence of chronic pain (Wiech and Tracey, 2009; Glattacker, Heyduck, and Meffert 2013; Ferreira-Valente, PaisRibeiro, and Jensen 2014). This could be addressed in further research by also including these factors. Additionally, regarding fear-avoidance only one study is present, meaning that also this factor could be of interest for further research as no conclusions can be drawn based on the results presented here.

A problem for the interpretation of the results of this review is the fact that the direction of the significant associations is in general very ambiguous. Further research could address this problem by making use of a novel, network-oriented, meta-analytic approach to characterize brain changes in chronic pain rather than isolated techniques (Cauda et al., 2014). This could lead to more straightforward results as more aspects are taken in mind in the analysis.

\section{Conclusion}

Pain catastrophizing is related to brain areas involved in pain processing, attention to pain, emotion and motor activity, and is associated with reduced engagement of the descending pain modulatory system. Evidence regarding fear-avoidance remains inconclusive and although evidence is in favour of no association between brain alterations and anxiety/depressive symptoms, there is some evidence for an association with increased brain activation in cognitive-affective areas.

Evidence on this subject is mainly limited to observational studies, which means that no causal interactions of the brain and maladaptive cognitive and emotional factors related to pain can be revealed. Hence, future research should investigate whether positively influencing these maladaptive cognitive and emotional factors (e.g. by therapy) can change these brain alterations in a positive way. Ideally, this should be investigated in a RCT, where this research question is addressed primarily and not as an additional or secondary aspect.

\section{Author contributions}

A.M. composed the methodology of the research, searched the databases, selected the eligible articles and made the initial draft of the manuscript. A.M. and I.C. performed the methodological quality check of the included papers, R.D.P. acted as third opinion in this process. All authors gave suggestions for the methodology of the search and helped in finalizing the manuscript in terms of grammar and content. All authors have read and approved the manuscript and have substantially contributed to the manuscript.

\section{References}

Alarcon, G., Cservenka, A., Rudolph, M.D., Fair, D.A., Nagel, B.J. (2015). Developmental sex differences in resting state functional connectivity of amygdala sub-regions. NeuroImage $115,235-244$.

Apkarian, A.V., Sosa, Y., Sonty, S., Levy, R.M., Harden, R.N., Parrish, T.B., Gitelman, D.R. (2004). Chronic back pain is associated with decreased prefrontal and thalamic gray matter density. J Neurosci 24 , 10410-10415.

Apkarian, A.V., Bushnell, M.C., Treede, R.D., Zubieta, J.K. (2005). Human brain mechanisms of pain perception and regulation in health and disease. Eur J Pain 9, 463-484.

Armijo-Olivo, S., Stiles, C.R., Hagen, N.A., Biondo, P.D., Cummings, G.G. (2012). Assessment of study quality for systematic reviews: A comparison of the cochrane collaboration risk of bias tool and the effective public health practice project quality assessment tool: Methodological research. J Eval Clin Pract 18, 12-18.

As-Sanie, S., Harris, R.E., Napadow, V., Kim, J., Neshewat, G., et al. (2012). Changes in regional gray matter volume in women with chronic pelvic pain: A voxel-based morphometry study. Pain 153, 1006-1014.

Baliki, M.N., Chialvo, D.R., Geha, P.Y., Levy, R.M., Harden, R.N., Parrish, T.B., Apkarian, A.V. (2006). Chronic pain and the emotional brain: Specific brain activity associated with spontaneous fluctuations of intensity of chronic back pain. J Neurosci 26, 1216512173. 
Baliki, M.N., Schnitzer, T.J., Bauer, W.R., Vania Apkarian, A. (2011). Brain morphological signatures for chronic pain. PLOS ONE 6, e26010.

Baliki, M.N., Mansour, A.R., Baria, A.T., Vania Apkarian, A. (2014). Functional reorganization of the default mode network across chronic pain conditions. PLOS ONE 9, e106133.

Barke, A., Baudewig, J., Schmidt-Samoa, C., Dechent, P., KronerHerwig, B. (2012). Neural correlates of fear of movement in high and low fear-avoidant chronic low back pain patients: An event-related fMRI study. Pain 153, 540-552.

Bigos, S.J., Battie, M.C., Spengler, D.M., Fisher, L.D., Fordyce, W.E., Hansson, T., Nachemson, A.L., Zeh, J. (1992). A longitudinal, prospective study of industrial back injury reporting. Clin Orthop Relat Res 279, 21-34.

Blankstein, U., Chen, J., Diamant, N.E., Davis, K.D. (2010). Altered brain structure in irritable bowel syndrome: Potential contributions of preexisting and disease-driven factors. Gastroenterology 138, 1783-1789.

Buckalew, N., Haut, M.W., Morrow, L., Weiner, D. (2008). Chronic pain is associated with brain volume loss in older adults: Preliminary evidence. Pain Med 9, 240-248.

Burgmer, M., Petzke, F., Giesecke, T., Gaubitz, M., Heuft, G., Pfleiderer, B. (2011). Cerebral activation and catastrophizing during pain anticipation in patients with fibromyalgia. Psychosom Med 73, 751-759.

Burton, A.K., Tillotson, K.M., Main, C.J., Hollis, S. (1995). Psychosocial predictors of outcome in acute and subchronic low back trouble. Spine 20, 722-728.

Cabral, D.M.C., Botelho Bracher, E.S., Prescatan Depintor, J.D., ElufNeto, J. (2014). Chronic pain prevalence and associated factors in a segment of the population of Sao Paulo city. Journal of Pain 15, 1081-1091.

Cauda, F., Palermo, S., Costa, T., Torta, R., Duca, S., Vercelli, U., Geminiani, G., Torta, D.M.E. (2014). Gray matter alterations in chronic pain: A network-oriented meta-analytic approach. Neuroimage Clin 4, 676-686.

Ceko, M., Catherine Bushnell, M., Fitzcharles, M.-A., Schweinhardt, P. (2013). Fibromyalgia interacts with age to change the brain. NeuroImage 3, 249-260.

Ceko, M., Gracely, J.L., Fitzcharles, M.-A., Seminowicz, D.A., Schweinhardt, P., Catherine Bushnell, M. (2015). Is a responsive default mode network required for successful working memory task performance? J Neurosci 35, 11595-11605.

Cifre, I., Sitges, C., Fraiman, D., Munoz, M.A., Balenzuela, P., Gonzalez-Roldan, A., Martinez-Jauand, M., Birbaumer, N., Chialvo, D.R., Montoya, P. (2012). Disrupted functional connectivity of the pain network in fibromyalgia. Psychosom Med 74, 55-62.

Crombez, G., Eccleston, C., Baeyens, F., Eelen, P. (1998). When somatic information threatens, catastrophic thinking enhances attentional interference. Pain 75, 187-198.

Diaz Mohedo, E., Warnberg, J., Baron Lopez, F.J., Mera Velasco, S., Cabello Burgos, A. (2014). Chronic pelvic pain in spanish women: Prevalence and associated risk factors. A cross-sectional study. Clin Exp Obstet Gynecol 41, 243-248.

Dureja, G.P., Jain, P.N., Shetty, N., Mandal, S.P., Prabhoo, R., et al. (2014). Prevalence of chronic pain, impact on daily life, and treatment practices in India. Pain Pract 14, E51-E62.

Elsenbruch, S., Rosenberger, C., Enck, P., Forsting, M., Schedlowski, M., Gizewski, E.R. (2010). Affective disturbances modulate the neural processing of visceral pain stimuli in irritable bowel syndrome: An fMRI study. Gut 59, 489-495.

Etkin, A., Wager, T.D. (2007). Functional neuroimaging of anxiety: A meta-analysis of emotional processing in PTSD, social anxiety disorder, and specific phobia. Am J Psychiatry 164, 1476-1488.

Ferreira-Valente, M.A., Pais-Ribeiro, J.L., Jensen, M.P. (2014). Associations between psychosocial factors and pain intensity, physical functioning, and psychological functioning in patients with chronic pain: a cross-cultural comparison. The Clinical Journal of Pain 30(8), $713-723$.

Foerster, B.R., Petrou, M., Edden, R.A.E., Sundgren, P.C., SchmidtWilcke, T., et al. (2012). Reduced insular gamma-aminobutyric acid in fibromyalgia. Arthritis Rheum 64, 579-583.
Geha, P.Y., Baliki, M.N., Norman Harden, R., Bauer, W.R., Parrish, T.B., Vania Apkarian, A. (2008). The brain in chronic CRPS pain: Abnormal gray-white matter interactions in emotional and autonomic regions. Neuron 60, 570-581.

Gerstner, G., Ichesco, E., Quintero, A., Schmidt-Wilcke, T. (2011). Changes in regional gray and white matter volume in patients with myofascial-type temporomandibular disorders: A voxel-based morphometry study. J Orofac Pain 25, 99-106.

Glass, J.M., Williams, D.A., Fernandez-Sanchez, M.-L., Kairys, A., Barjola, P., Heitzeg, M.M., Clauw, D.J., Schmidt-Wilcke, T. (2011). Executive function in chronic pain patients and healthy controls: Different cortical activation during response inhibition in fibromyalgia. J Pain 12, 1219-1229.

Glattacker, M., Heyduck, K., Meffert, C. (2013). Illness beliefs and treatment beliefs as predictors of short-term and medium-term outcome in chronic back pain. Journal of Rehabilitation Medicine 45(3), 268-276.

Gracely, R.H., Geisser, M.E., Giesecke, T., Grant, M.A.B., Petzke, F., Williams, D.A., Clauw, D.J. (2004). Pain catastrophizing and neural responses to pain among persons with fibromyalgia. Brain 127, 835-843.

Gupta, A., Rapkin, A.J., Gill, Z., Kilpatrick, L., Fling, C., et al. (2015). Disease-related differences in resting-state networks: A comparison between localized provoked vulvodynia, irritable bowel syndrome, and healthy contrlo subjects. Pain 156, 809-819.

Gustin, S.M., Peck, C.C., Wilcox, S.L., Nash, P.G., Murray, G.M., Henderson, L.A. (2011). Different pain, different brain: Thalamic anatomy in neuropathic and non-neuropathic chronic pain syndromes. J Neurosci 31, 5956-5964.

Hauser, W., Wolfe, F., Henningsen, P., Schmutzer, G., Brahler, E., Hinz, A. (2014). Untying chronic pain: Prevalence and societal burden of chronic pain stages in the general population - A cross-sectional survey. BMC Public Health 14, 352.

Hogstrom, L.J., Westlye, L.T., Walhovd, K.B., Fjell, A.M. (2013). The structure of the cerebral cortex across adult life: Age-related patterns of surface area, thickness, and gyrification. Cereb Cortex 23, 25212530 .

Hong, J.-Y., Kilpatrick, L.A., Labus, J., Gupta, A., Jiang, Z., et al. (2013). Patients with chronic visceral pain show sex-related alterations in intrinsic oscillations of the resting brain. J Neurosci 33, 11994-12002.

Hsu, M.C., Harris, R.E., Sundgren, P.C., Welsh, R.C., Fernandes, C.R. Clauw, D.J., Williams, D.A. (2009). No consistent difference in gray matter volume between individuals with fibromyalgia and agematched healthy subjects when controlling for affective disorder. Pain $143,262-267$

Ichesco, E., Schmidt-Wilcke, T., Bhavsar, R., Clauw, D.J., Peltier, S.J., et al. (2014). Altered resting state connectivity of the insular cortex in individuals with fibromyalgia. J Pain 15, 815-826.e1.

Jackson, N., Waters, E., for the Guidelines for Systematic Reviews in Health Promotion and Public Health Taskforce. (2005). Criteria for the systematic review of health promotion and public health interventions. Health Promot Int 20, 367-374.

Jackson, T., Chen, H., Iezzi, T., Yee, M., Chen, F. (2014). Prevalence and correlates of chronic pain in a random population study of adults in Chongqing, China. Clin J Pain 30, 346-352.

Jensen, M.P., Ehde, D.M., Hoffman, A.J., Patterson, D.R., Czerniecki, J.M., Robinson, L.R. (2002). Cognitions, coping and social environment predict adjustment to phantom limb pain. Pain 95, 133142 .

Jensen, K.B., Kosek, E., Wicksell, R., Kemani, M., Olsson, G., Merle, J.V., Kadetoff, D., Ingvar, M. (2012). Cognitive behavioral therapy increases pain-evoked activation of the prefrontal cortex in patients with fibromyalgeia. Pain 153, 1495-1503.

Khan, S.A., Keaser, M.L., Meiller, T.F., Seminowicz, D.A. (2014). Altered structure and function in the hippocampus and medial prefrontal cortex in patients with burning mouth syndrome. Pain $155,1472-1480$.

Kim, J.-Y., Kim, S.-H., Seo, J., Kim, S.-H., Han, S.W., et al. (2013). Increased power spectral density in resting-state pain-related brain networks in fibromyalgia. Pain 154, 1792-1797. 
Kucyi, A., Moayedi, M., Weissman-Fogel, I., Goldberg, M.B., Freeman B.V., Tenenbaum, H.C., Davis, K.D. (2014). Enhanced medial prefrontal-default mode network functional connectivity in chronic pain and its association with pain rumination. J Neurosci 34, 39693975

Lieberman, G., Shpaner, M., Watts, R., Andrews, T., Filippi, C.G., Davis, M., Naylor, M.R. (2014). White matter involvement in chronic musculoskeletal pain. J Pain 15, 1110-1119.

Lloyd, D., Findlay, G., Roberts, N., Nurmikko, T. (2008). Differences in low back pain behavior are reflected in the cerebral response to tactile stimulation of the lower back. Spine 33, 1372-1377.

Lloyd, D.M., Findlay, G., Roberts, N., Nurmikko, T. (2014). Illness behavior in patients with chronic low back pain and activation of the affective circuitry of the brain. Psychosom Med 76, 413-421.

Loggia, M.L., Berna, C., Kim, J., Cahalan, C.M., Martel, M.-O., et al. (2015). The lateral prefrontal cortex mediates the hyperalgesic effects of negative cognitions in chronic pain patients. J Pain 16, 692-699.

Lutz, J., Jaeger, L., de Quervain, D., Krauseneck, T., Padberg, F., et al. (2008). White and gray matter abnormalities in the brain of patients with fibromyalgia a diffusion-tensor and volumetric imaging study. Arthritis Rheum 58, 3960-3969.

Malfliet, A., Kregel, J., Cagnie, B., Kuipers, M., Dolphens, M., et al. (2015). Lack of evidence for central sensitization in idiopathic, nontraumatic neck pain: A systematic review. Pain Physician 18, 223-236.

Melzack, R., Wall, P.D. (1982). The Challenge of Pain. (New York: Basic Books).

Moher, D., Liberati, A., Tetzlaff, J., Altman, D.G. (2009). Preferred reporting items for systematic reviews and meta-analyses: The PRISMA statement. Ann Intern Med 151, 264-269, W64.

Obermann, M., Rodriguez-Raecke, R., Naegel, S., Holle, D., Mueller, D., et al. (2013). Gray matter volume reduction reflects chronic pain in trigeminal neuralgia. NeuroImage 74, 352-358.

Riederer, F., Landmann, G., Gantenbein, A.R., Stockinger, L., Egloff, N., et al. (2015). Nondermatomal somatosensory deficits in chronic pain are associated with cerebral grey matter changes. World $\mathrm{J}$ Biol Psychiatry, 1-12.

Riskowski, J.L. (2014). Associations of socioeconomic position and pain prevalence in the United States: Findings from the National Health and Nutrition Examination Survey. Pain Med 15, 1508-1521.

Scheinost, D., Finn, E.S., Tokoglu, F., Shen, X., Papademetris, X., Hampson, M., Todd Constable, R. (2015). Sex differences in normal age trajectories of functional brain networks. Hum Brain Mapp 36, 1524-1535.

Schmidt-Wilcke, T. (2015). Neuroimaging of chronic pain. Best Pract Res Clin Rheumatol 29, 29-41.
Schmidt-Wilcke, T., Luerding, R., Weigand, T., Jurgens, T., Schuierer, G., Leinisch, E., Bogdahn, U. (2007). Striatal grey matter increase in patients suffering from fibromyalgia-a voxel-based morphometry study. Pain 132(Suppl), S109-S116.

Seminowicz, D.A., Wideman, T.H., Naso, L., Hatami-Khoroushahi, Z., Fallatah, S., et al. (2011). Effective treatment of chronic low back pain in humans reverses abnormal brain anatomy and function. $J$ Neurosci 31, 7540-7550.

Seminowicz, D.A., Shpaner, M., Keaser, M.L., Michael Krauthamer, G., Mantegna, J., et al. (2013). Cognitive-behavioral therapy increases prefrontal cortex gray matter in patients with chronic pain. J Pain 14, 1573-1584.

Thomas, B.H., Ciliska, D., Dobbins, M., Micucci, S. (2004). A process for systematically reviewing the literature: Providing the research evidence for public health nursing interventions. Worldviews Evid Based Nurs 1, 176-184.

Valet, M., Gundel, H., Sprenger, T., Sorg, C., Muhlau, M., Zimmer, C., Henningsen, P., Tolle, T.R. (2009). Patients with pain disorder show gray-matter loss in pain-processing structures: A voxel-based morphometric study. Psychosom Med 71, 49-56.

Vehof, J., Zavos, H.M.S., Lachance, G., Hammond, C.J., Williams, F.M.K. (2014). Shared genetic factors underlie chronic pain syndromes. Pain 155, 1562-1568.

Wiech, K., Tracey, I. (2009). The influence of negative emotions on pain: Behavioral effects and neural mechanisms. Neurolmage 47, 987-994.

Wiech, K., Ploner, M., Tracey, I. (2008). Neurocognitive aspects of pain perception. Trends Cogn Sci 12, 306-313.

Xu, C., Li, C., Hongli, W., Yuanyuan, W., Sheng, H., Zhu, Y., et al. (2015). Gender differences in cerebral regional homogeneity of adult healthy volunteers: A resting-state FMRI study. Biomed Res Int 2015, 183074.

Yu, R., Gollub, R.L., Spaeth, R., Napadow, V., Wasan, A., Kong, J. (2014). Disrupted functional connectivity of the periaqueductal gray in chronic low back pain. NeuroImage 6, 100-108.

\section{Supporting Information}

Additional Supporting Information may be found online in the supporting information tab for this article:

Appendix S1. Entry of search terms.

Table S2. Level of evidence.

Table S3. Level of conclusion. 